NASA Technical Memorandum 105291

AIAA-91-1999

\title{
Linear Quadratic Servo Control of a Reusable Rocket Engine
}

Jeffrey L. Musgrave

Lewis Research Center

Cleveland, Ohio

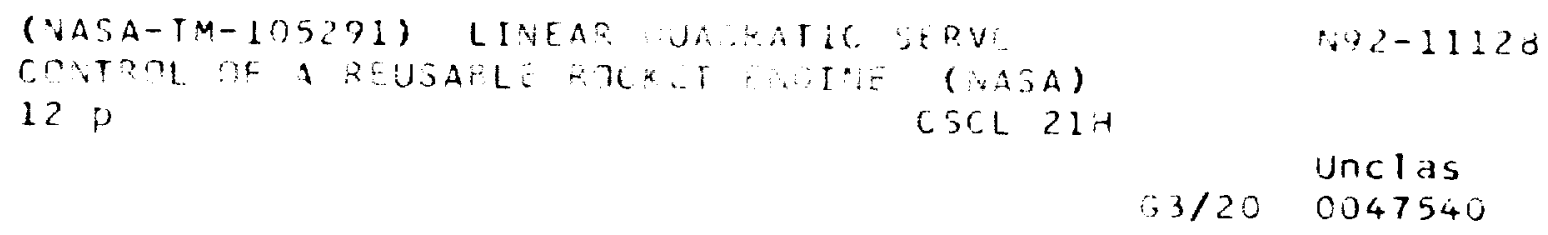

Prepared for the 27th Joint Propulsion Conference cosponsored by AIAA, SAE, ASME, and ASEE Sacramento, California, June 24-27, 1991 



\title{
LINEAR QUADRATIC SERVO CONTROL OF A REUSABLE ROCKET ENGINE*
}

\author{
Jeffrey L. Mlusgrave \\ National Aeronanlics and Space Administration \\ I.ewis Research Center \\ Cleveland, Ohio 441.35
}

\begin{abstract}
$\underline{\text { Abstract }}$
This work develops a new design method for the Servo Compensator in the frequency domain using singular values and applies the method to a reusable rocket engine. An Intelligent Control System for reusable rocket engines has been proposed which includes a diagnostic system, a control system and an intelligent coordinator which determines engine control strategies based on the identified failure modes. The method provides a means of generating various linear multivariable controllers capable of meeting performance and robustness specifications and accommodating failure modes identified by the diagnostic system. Command following with set point control is necessary for engine operation. A Kalman filter reconstructs the state while Loop transfer recovery recovers the required degree of robustness while maintaining satisfactory rejection of sensor noise from the command error. The approach is applied to the design of a controller for a rocket engine satisfying performance constraints in the frequency domain. Simulation results demonstrate the performance of the linear design on a nonlinear engine model over all power levels during mainstage operation.
\end{abstract}

\section{Introduction}

An Intelligent Control System (ICS) for reusable rocket engines has been proposed ${ }^{1}$ for the purpose of widening the range of operation, enhancing overall engine performance and reducing the amount of required maintenance. Improvements in engine durability through control of critical temperatures and pressures should improve the usable engine life without sacrificing the enormous thrust levels required for mission success. The ICS is composed of a real-time diagnostic system, a multivariable controller and an "intelligent coordinator". The diagnostic system identifies engine failure modes including actuator and sensor failures online and supplies this information to the coordinator. The coordinator selects the best possible control strategy based on the severity of the failure and provides this data to the reconfigurable controller. The controller implements the strategy based upon a priori design criteria in order to balance the inherent tradeoff between engine performance and engine life. The servomechanism approach ${ }^{2}$ is useful for solving such a problem. Soft failures such as a drop in turbine efficiency may be accommodated by changing the set points of controlled engine variables. Hard failures such as sensors out of range or sticking actuators (valves) will require reconfiguration of the controller based on the particular component and its role in the closed loop system. Degradations in other engine components may be accommodated by adding and/or removing variables from the set of controlled quantities and determining new set points. Command following is required because transitions between set points must not result in stress cycles which adversely affect the durability of the engine. This work develops a new design method in the frequency domain for the Servo Compensator ${ }^{4}$ which meets the requirements for an ICS controller.

Using the linear quadratic regulator for the design of a command following controller was first introduced by Athans $^{3}$. Davison and Goldenberg ${ }^{4}$ use state augmentation in a similar manner to synthesize the robust Servo Compensator possessing certain degrees of robustness to variations in the plant model based on the availability of outputs for measurement. The method relies primarily on augmenting the state vector of the plant with an internal model of the plant disturbances and reference commands resulting in a multivariable compensator with a stabilizing loop and a feed-forward loop. Davison ${ }^{5}$ proposed a technique for constructing the gains for the Servo Compensator for the case where the plant model is unknown. Wang and Munro extend earlier results by demonstrating how the Servo Compensator can be used with linear quadratic regulator theory to synthesize controller gains for both step and ramp disturbances and input commands.

Much emphasis has been placed on guaranteed robustness (ability for a control system to maintain stability given uncertainty in the design model) in linear control systems over the past decade and many excellent papers treating the subject permeate the literature. The seminal work of Doyle and Stein ${ }^{7}$ made clear the notion that the design of a Kalman filter for state estimation in

"Copyright (1) 1991 by the AIAA, Inc. No copyright is asserted in the U.S. under Title 17, US Code. The U.S. Govemment has a royalty-free license to exercise all rights under the copyright claimed herein for Governmental purposes. All other rights are reserved by the copyright owner. 
a linear state feedback controller can destroy the guaranteed robustness associated with the quadratic regulator if such a simple criterion as "the filter poles must be sufficiently faster than the closed loop system poles" guides the design process. As a result, preservation of controller robustness complicates compensator design for linear closed loop feedback control.

A majority of the analysis procedures for determining the degree of robustness in a closed loop control system are typically specified in the frequency domain using singular values of the sensitivity $(S(s)=$ $\left.[\mathrm{I}+\mathrm{G}(\mathrm{s}) \mathrm{K}(\mathrm{s})]^{-1}\right)$ and complementary sensitivity $(\mathrm{T}(\mathrm{s})=$ $\left.G(s) K(s)[1+G(s) K(s)]^{-1}\right)$ functions as a measure of worst case behavioo $8,9,10$. In particular, the LQG/LTR methodology $y^{11.12}$ employs these ideas directly in the design and analysis of a dynamic compensator using loop shaping ideas in the frequency domain on transfer functions created by breaking loops at physical points designated by XX's in fig. 1 . Athans and Stein $^{13}$ first discussed the design of the servo using frequency domain techniques based on transfer functions consistent with the ideas presented by Doyle and Stein. The results presented here extend those of Athans and Stein into a complete design methodology in the frequency domain using singular value inequalities and loop transfer recovery (LTR).

This paper presents a general methodology used to design a class of linear multivariable controllers for an ICS. A servomechanism approach allows set point control with command following. The design methodology is presented along with an analysis of the performance and robustness characteristics. Estimator design is performed in the framework of the Kalman filter formalism with emphasis on using a sensor set different from the commanded variables. Loop transfer recovery modifies the nominal plant noise intensities to obtain the desired degree of robustness to uncertainty reflected at the plant input. Finally, an example demonstrates the technique in the design of a controller which will be used in an ICS for a reusable rocket engine.

\section{Servo Compensator with LTR}

Consider a proper linear time invariant system written in state space form as

$$
\begin{aligned}
& \dot{x}(t)=A x(t)+B u(t)+\zeta(t) \\
& y(t)=C x(t) \\
& z(t)=H x(t)+\Gamma \eta(t)
\end{aligned}
$$

where $x \in \Re^{n}, u \in \Re^{m}, z \in \Re^{m}$, and $y \in \Re^{r}$ with matrices $A, B, C, H$, and $\Gamma$ all of appropriate dimension with $m \geq r$. Additionally, $\zeta(t)$ and $\eta(t)$ are zero mean vector random processes with nomal distributions with intensities $Q_{p}=Q_{p}{ }^{\top} \in \Re^{n \times n}$ and $Q_{p} \geq 0$, and $R_{1}=R_{d}{ }^{T} \in$ $\Re^{m \times m}$ and $R_{1}>0$ respectively. Here, $y(t)$ and $r(t)$ represent commanded quantities and reference commands, respectively. The measurements $z(t)$ are used for state reconstruction via the Kalman filter. To achieve arbitrary set point control with integral action and tracking of commands, a control law of the form

$$
u(t)=-K_{s p} x(t)-K_{t} \int_{0}^{t} e(\tau) d \tau
$$

is desired, where $e(t)=r(t)-y(t), K_{\text {op }} \in \Re^{m \times n}, K_{i} \in$

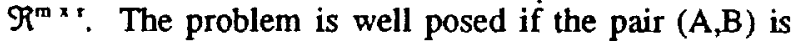
stabilizable, if $(\mathrm{H}, \mathrm{A})$ and $(\mathrm{C}, \mathrm{A})$ are detectable, and if transmission zeros of $\{A, B, C\}$ do not appear at the origin.

$\mathrm{K}_{\mathrm{ap}}$ and $\mathrm{K}_{\mathrm{i}}$ are determined by augmenting the state with

$$
e_{a}(t)=\int_{0}^{t}[y(\tau)-r(\tau)] d \tau
$$

giving

$$
\frac{d}{d t}\left[\begin{array}{l}
x(t) \\
e_{a}(t)
\end{array}\right]=\left[\begin{array}{ll}
A & 0 \\
C & 0
\end{array}\right]\left[\begin{array}{l}
x(t) \\
e_{a}(t)
\end{array}\right]+\left[\begin{array}{l}
B \\
0
\end{array}\right] u(t)
$$

when $r(t)$ is zero. The synthesis problem may be solved by minimizing a standard quadratic cost function

$$
\frac{1}{2} \int_{0}^{\infty}\left[x(\tau) e_{a}(\tau)\right] Q_{a}\left[\begin{array}{l}
x(\tau) \\
e_{a}(\tau)
\end{array}\right]+u(\tau)^{T} R u(\tau) d \tau
$$

where $R=R^{T}>0$ and $Q_{a}$ is typically taken to be

$$
Q_{a}=\left[\begin{array}{ll}
0 & 0 \\
0 & Q
\end{array}\right]
$$

with $Q=Q^{T}>0$. The detectability of $(C, A)$ is required due to the special structure of the weighting matrix $Q$. for the augmented system. The linear state feedback control satisfying this minimization problem can be obtained from the solution P of an Algebraic Ricatti Equation (ARE) such that

$$
\left[\begin{array}{ll}
K_{s p} & K_{i}
\end{array}\right]=R^{-1}\left[\begin{array}{l}
B \\
0
\end{array}\right]^{T} P
$$

The design reduces to the selection of the matrices $Q$ and $R$ satisfying performance and robustness constraints prescribed for the closed loop system. 


\section{$\underline{\text { Servo Compensator Design }}$}

In order to design a controller with good command following, and robustness to modelling errors and plant disturbances, the singular values of two different transfer functions must be designed simultaneously ${ }^{13}$. Fig. 2 shows the Servo Compensator configuration with full state feedback. The design of a controller with good command following properties requires $R(s)$ equal to $Y(s)$ over the range of frequencies contained in typical inputs. For design purposes, $Y(s)$ may be written as

$$
Y(s)=\left[\begin{array}{c}
\left.I+C \Phi B\left(I+K_{s} \Phi B\right)^{-1} G_{y}(s)\right]^{-1} C \Phi B(I+ \\
\left.K_{s} \Phi B\right)^{-1} G_{y}(s) R(s)
\end{array}\right.
$$

where $\mathrm{R}(\mathrm{s}) \in C^{\mathrm{m}}, \Phi=(\mathrm{sI}-\mathrm{A})^{-1}$ and, $\mathrm{G}_{\mathrm{y}}(\mathrm{s})=\mathrm{K}_{\mathrm{f}}(\mathrm{sI})^{-1}$. Good command following requires

$$
\underline{g}\left[C \Phi B\left(I+K_{s p} \Phi B\right)^{-1} G_{y}(s)\right]>>1
$$

where $\underline{\sigma}()$ represents the minimum singular value which follows immediately from the properties of singular values $^{12}$. The transfer function appearing in (2) corresponds to breaking the error loop at the XX's shown at point 2 in fig. 2 and synthesizing the open loop transfer function from $E^{\prime}(s)$ to $E^{\prime \prime}(s)$ with $R(s)$ equal to zero. The singular values may be altered directly by modifying the weights in the solution of the ARE until the minimum singular value bas satisfactory gain over all frequencies of interest. Recall that singular values represent sufficient conditions since they specify worst case directions on a multivariable transfer function.

The controller should also possess a certain degree of robustness to low frequency modelling errors as noted in fig. 2 by $D(s)$. In particular, the effect of $D(s)$ on $E(s)$ should be made as small as possible. To determine the requirement explicitly, E(s) may be written as

$$
E(s)=-\left[I+C \Phi B\left(I+K_{s p} \Phi B\right)^{-1} G_{y}(s)\right]^{-1} D(s)
$$

which shows that the condition specified in (2) for good command following is sufficient to guarantee disturbance rejection resulting from low frequency modelling errors as in the traditional LQG setup shown in fig. 1.

The final issue in the servo design involves minimizing the impact of modelling errors reflected at the plant input by considering the physical point at 1 in fig 2. Additionally, the effect of any sensor noise on the command channel can be included with "modelling errors" as with any noise on the channel connecting the controller to the plant. The control input equation may be written as

$$
U(s)=-K_{\nexists p} X(s)+G_{y}(s)(R(s)-Y(s))-K_{\Phi} \delta X(s)-G_{y}(s) \delta Y
$$

where $\delta \mathrm{X}(\mathrm{s})$ and $\delta \mathrm{Y}(\mathrm{s})$ represent any lumped additive uncertainty associated with modelling error and sensor noise. Taking $R(s)$ equal to zero and letting $D_{m}(s)$ represent both plant uncertainties and noise disturbances, the equation may be rewritten as

$$
U(s)=\left(I+K_{s p} \Phi B+G_{y}(s) C \Phi B\right)^{-1} D_{m}(s)
$$

illustrating that disturbance rejection at the input corresponds to selecting the regulator weights such that

$$
\underline{\sigma}\left(K_{s p} \Phi B+G_{y}(s) C \Phi B\right)>>1
$$

over the frequencies where $D_{m}(s)$ has the majority of its energy. It can be seen from fig. 3 that the transfer function in (3) results from breaking the loop at the physical point 1 denoted by XX's and synthesizing the relation from U'(s) to U' (s) with $R(s)$ equal to zero.

In summary, the design of the Servo Compensator consists of selecting $Q$ and $R$ to satisfy the inequalities given in (2) and (3). The design requires specifications for performance and robustness in the frequency domain which adequately represent the desired characteristics for the closed loop system ${ }^{14}$.

\section{Estimator Design}

A Kalman filter may used to realize the above full state feedback controller since all plant states will not be measurable in general. The estimator must not destroy the command following (performance) or robustness properties of the controller while still possessing the desired amount of filtering of measurement noise. Singular values may be used to determine the extent of sensor noise rejection in a worst case sense by examining the effect of noise on the error $E(s)$.

With LQG, sensor noise can be rejected from the error equation ${ }^{12}$ by satisfying

$$
\bar{\sigma}(G(s) K(s))<<1
$$

where $\mathrm{G}(\mathrm{s}) \mathrm{K}(\mathrm{s})$ results from breaking the loop at the $X X$ 's at the physical point 2 in fig. 1 and constructing the transfer function from $Y^{\prime}(s)$ to $Y^{\prime \prime}$ '(s) with $R(s)=0$. Employing a similar argument here and breaking the loop at the XX's at physical point 3 in fig. 3 gives 


$$
\begin{aligned}
Z^{\prime \prime}(s) & =-H \Phi B\left(I+G_{y}(s) C \Phi B\right)^{-1} K_{\neq p}\left(I+\Phi K_{f} H\right. \\
& +\Phi B\left(I+G_{y}(s) C \Phi B\right)^{-1} K_{s p} J^{-1} \Phi K_{z} Z^{\prime}(s)
\end{aligned}
$$

which should be sufficient to describe the effect of sensor noise on $E(s)$. However, from fig. 3 the transfer function from $\eta(s)$ to $E(s)$ with $R(s)$ equal to zero is

$$
\begin{gathered}
E(s)=C \Phi B\left(I+G_{y}(s) C \Phi B\right)^{-1} K_{s p}\left[I+\Phi K_{d} H+\Phi(B+\right. \\
K, H \Phi B)\left(I+G_{y}(s) C \Phi B\right)^{-1} K_{s p} J^{-1} \Phi K_{.} \eta(s)
\end{gathered}
$$

which is different from (5). Moreover, if $\mathrm{C}$ is identical to $\mathrm{H}$ as in many applications, (6) includes an additional term in the "denominator" which invalidates the usage of (5) for (6). Consequently, (6) must be used directly by employing the standard analysis with singular values as a worst case measure. The impact of sensor noise on the error can be minimized by making

$$
\begin{aligned}
& \bar{\sigma}\left(C \Phi B ( I + G _ { y } ( s ) C \Phi B ) ^ { - 1 } K _ { s p } \left(I+\Phi K_{e} H+\Phi(B\right.\right. \\
& \left.\left.\left.+K_{e} H \Phi B\right)\left(I+G_{y}(s) C \Phi B\right)^{-1} K_{s p}\right)^{-1} \Phi K_{e}\right)<1
\end{aligned}
$$

over frequencies where $\eta(s)$ has its energy. If the designer is unwary and uses (5) as the criterion in a singular value analysis the resulting filter will not possess the expected noise rejection properties.

The estimator design may be approached by selecting some nominal process noise intensity for $Q_{p}$ (typically $B^{\top}$ ) and choosing $R_{t}=p \Gamma$ where $p$ is some scalar parameter. The tuning parameter $p$, can be increased or decreased such that the resulting Kalman gain $\left(\mathrm{K}_{\mathrm{e}}\right)$ satisfies $(7)$.

\section{Loop Transfer Recovery}

Loop transfer recovery in conjunction with the Kalman filter allows the designer to perform a tradeoff between the amount of sensor noise rejection and robustness to unmodeled dynamics inherent in a standard LQG controller via a single scalar parameter in the ARE for the estimator or equivalently, the regulator design". In this work, the estimator will be used to recover a certain degree of robustness in the Servo Compensator while maintaining an acceptable level of noise rejection from the commanded error equation for the case when commanded outputs are not used for state reconstruction. Additionally, introduction of the estimator does not adversely affect the command following performance which has been designed into the servo by satisfying (2) over the frequencies in typical inputs.

To determine the impact of the estimator on robustness, the estimator equation may be written from fig. 4 with the loop broken at the XX's at point 1 as

$$
\hat{X}(s)=\Phi\left(B U^{\prime \prime}(s)+K_{\triangleleft} H \Phi B U^{\prime}(s)\right)-\Phi K_{\triangleleft} H \hat{X}(s) .
$$

Rearranging terms and applying an identity gives

$$
\begin{aligned}
& \hat{X}(s)=\Phi\left[B(H \Phi B)^{-1}-K_{0}\left(I+H \Phi K_{e}\right)^{-1}\right] H \Phi B U^{\prime \prime}(s)+ \\
& \Phi K_{a}\left(I+H \Phi K_{\alpha}\right)^{-1} H \Phi B U^{\prime}(s) \text {. }
\end{aligned}
$$

from which it follows that

$$
B(H \Phi B)^{-1}=K_{\triangleleft}\left(I+H \Phi K_{\downarrow}\right)^{-1}
$$

will remove the estimator from the loop making

$$
X(s)=\hat{X}(s)=\Phi B U^{\prime}(s) \text {. }
$$

It is important to note the existence of the inverse of $\mathrm{H} \Phi \mathrm{B}$ is assumed over all frequency for LTR to be applicable with the Kalman filter.

Command following properties for the Servo Compensator are invariant to the introduction of the state estimator. This may be seen by rewriting the estimation equation after breaking the loop at the XX's designated by point 2 in fig. 3 as

$$
s \hat{X}(s)=A \hat{X}(s)+B U(s)+K_{e}(Z(s)-H \hat{X}(s))
$$

which simplifies by substituting for $Z(s)$ and collecting like terms to yield

$$
\hat{X}(s)=\left(\Phi^{-1}+K_{e} H\right)^{-1}\left(I+K_{e} H \Phi\right) B U(s) .
$$

The transfer function from $E^{\prime}(s)$ to $E^{\prime \prime}(s)$ can now be written as

$$
E^{\prime \prime}(s)=-C \Phi B\left(I+K_{s p} \Phi B\right)^{-I} G_{y}(s) E^{\prime}(s)
$$

which is identical to the full state feedback case. One of the strengths of the Servo Compensator approach is the estimator dynamics do not degrade the "steady-state" command following performance designed into the compensator using (2).

Doyle and Stein proposed a general method for modifying the Kalman filter gain to achieve LTR pointwise in " $\mathrm{s"}$ as a function of a single scalar parameter. In particular, let $Q_{p}$ and $R$, be the nominal noise intensities for the standard filter problem as determined in the previous section, the proposed modification procedure appears as

$$
Q=Q_{p}+q^{2} B V B^{T}
$$

where $\mathrm{V} \geq 0$ is some weighting matrix selected by the designer. Matson and Maybeck have recently proven ${ }^{15}$ that (8) will be satisfied in the limit as q gets arbitrarily large.

Achieving LTR by way of the Kalman filter results in an important restriction on the realization $\{A, B, H\}$. If $\mathrm{K}_{\mathrm{e}}$ is the Kalman filter gain, then as q gets arbitrarily large the filter poles approach the plant transmission zeros or their stable images. LTR is essentially the process of canceling the plant zeros with the filter poles. However, the Kalman filter synthesis will map the plant's non-minimum phase zeros be they finite or infinite, to their respective stable images making LTR impossible. Several authors including Stein and Athans" address the issue of LTR for plants with non- 
minimum phase zeros.

The previous discussion highlights some of the difficulties in using LTR. However, these can be avoided in the Servo Compensator setup since $\mathrm{H}$ may be selected independently of $C$ which results from the control objective. That is, for a given objective $\{A, B, C\}$ may not be minimum phase and/or invertible. However, $H$ may be selected independently of the objective based on the available measurements such that the requirements for LTR are satisfied. Additionally, in an environment where a number of control strategies (via the $\mathrm{C}$ matrix) may be used by reconfiguring the controller, a sensor set $(\mathrm{H})$ which is invariant simplifies the overall compensator design since the nominal estimator portion of the design is done only once.

\section{Design Example}

This paper develops a design method suited to the purpose of controller synthesis for an Intelligent Control System. The controller must perform over all power levels during mainstage operation, provide command following with set point control, be multi-objective, perform over a variety of engine builds based on a nominal model, and be insensitive to component wear during operation. These five requirements cover the range of abilities for a controller in a rocket engine ICS $^{1}$. The focus of the present design is for mainstage operation defined from start plus five seconds to shutdown. Any time less than start plus five seconds is considered to be the startup. Control of the startup transients could improve the durability of the engine. However, the approach developed here cannot be used directly since the dynamics during this period are not well understood making the synthesis of appropriate $\{A, B, C\}$ difficult.

\section{Space Shuttle Main Engine (SSME)}

The SSME is the first large reusable rocket engine using a staged combustion cycle with two high pressure turbopumps (oxygen and hydrogen) fed by two low pressure turbopumps. Fig. 4 provides a schematic of a modified SSME with an additional actuator. The modified SSME contains the five original actuators; Fuel Preburner Oxidizer Valve (FPOV), Oxidizer Preburner Oxidizer Valve (OPOV), Main Oxidizer Valve (MOV), Main Fuel Valve (MFV), and Coolant Control Valve (CCV). The current control system for the SSME uses FPOV and OPOV for mixture ratio (MR) and thrust control via chamber pressure $(\mathrm{Pc})$, respectively. An additional valve called the Oxidizer Preburner Fuel Valve (OPFV) is added to give control authority over the temperatures in the high pressure turbines. The location of these valves with respect to the turbomachinery and combustion chamber may seen in fig. 4. The engine is fully throttlable during mainstage from $65 \%$ through $109 \%$ rated power with a rate limit on thrust commands of $10 \%$ power per second.

The proposed control design is targeted for demonstration on the Technology Test Bed where a large number of sensors are available for the purpose of feedback. For this study, ten measurements are selected with locations on the engine as shown in fig. 4 . In particular, Pfdl and Tfdl are the discharge pressure and temperature of the low pressure fuel turbopump, Qffm is the volumetric flow into the high pressure fuel turbopump, Tft2d and Tot $2 \mathrm{~d}$ are the discharge temperatures of the high pressure fuel and lox turbines, $\mathrm{Pc}$ is the pressure in the main combustion chamber, $\mathrm{P4}$ and P5 are the pressures in the nozzle and main combustion chamber cooling jackets, $\mathrm{P} 9$ is the pressure of fuel supply to the preburners, and Pfd2 is the discharge pressure from the high pressure fuel turbopump.

A simplified nonlinear simulation of the engine ${ }^{14}$ in Matrix $_{x}$ System Build ${ }^{\mathrm{TM}}$ is used for the purpose of linear model generation and control design. The engine model contains thirty nine states based primarily on flow continuity equations. Hydrogen and oxygen properties are contained in look-up tables as well as hydrogen combustion properties for use in the prebumers and main combustion chamber. The additional actuator OPFV is modeled after OPOV having second order dynamics and backlash and stiction. Each of the six actuators is modeled using four states. Linear perturbation state-space models are generated at the $65 \%, 80 \%$ and $100 \%$ power levels without actuator dynamics for the purpose of control design. However, preliminary investigation confirms that a $100 \%$ power design model is sufficient for mainstage operation making gain scheduling on power unnecessary.

\section{Compensator Design}

Since the engine is open loop stable, the primary purpose of the controller is to provide multiple power levels while maintaining the temperature in the main combustion chamber at or near the design point. Thrust is not a measurable quantity, but controlling $\mathrm{Pc}$ is sufficient to regulate thrust. Combustion temperature can be controlled by MR for any level of thrust. However, MR is not measurable and must be estimated using Pfdl, Tfdl, Pc and Qffm. The high pressure turbine discharge temperatures (Tft2d and Tot $2 \mathrm{~d}$ ) are controlled to minimize temperature excursions which result in turbine blade fatigue. An additional benefit of controlling turbine temperatures can be illustrated by the following example. As the engine operates, turbine efficiency decreases resulting in higher turbine discharge temperatures to maintain thrust requirements. A 
large decrease in turbine efficiency can lead to temperature redlines. By controlling both thrust and turbine temperatures, the controller is able to tradeoff increasing turbine temperatures with decreasing thrust thereby avoiding an engine cutoff. For the present example, the controlled quantities are given by $y=[\mathrm{Pc}$ MR Tf2d Tot2d] ${ }^{\mathrm{T}}$.

The first step in the design is the synthesis of the controller gains using regulator theory. In particular, diagonal matrices $Q \in \mathfrak{F}^{\times \times}$and $R \in \mathfrak{K}^{\mathrm{m} \times \text { m }}$ must be chosen to satisfy eqs. (2) and (3) over a specified frequency range. Thrust rates are limited on the SSME in the frequency domain by choosing $Q$ and $R$ such that eq. (2) is satisfied up to 1.5 radians per second. Additionally, eq. (3) must be satisfied over as broad a range as possible for robustness. The results of the full state feedback servo design are summarized in figs. 5 and 6 . The solid line in fig. 5 provides a measure of the robustness in the controller with full state feedback while fig. 6 shows that command following constraints are met for the specified frequency range. Analysis of loop shapes using singular values can be performed only after scaling of the physical quantities at the input and output of the transfer function has been performed to allow relative comparisons.

The selection $z=\left[\mathrm{P} 5 \mathrm{Pfd} 2 \mathrm{Tft} 2 \mathrm{~d}\right.$ Tot2d P4 P9 ${ }^{\mathrm{T}}$ for state reconstruction results in a minimum phase and invertible realization $\{A, B, H\}$. The objective of the nominal design is to choose $\mathrm{Q}_{\mathrm{p}}=\mathrm{q}^{2} \mathrm{BVB}^{\mathrm{T}}$ and $\mathrm{R}_{\mathrm{s}}=\mathrm{p} \Gamma$ such that eq. (7) is satisfied over all frequency. A nominal design using $q^{2}=0.00022$ and $p=5 . \times 10^{6}$ gave the singular value plots for sensor noise rejection shown in fig. 7. However, fig. 5 shows how the introduction of the estimator degrades the robustness of the controller by lowering the minimum singular value of eq. (3). LTR can be performed by increasing $q$ in the estimator design to recover the robustness inherent in the full state feedback compensator as shown in fig. 5 with $q^{2}=0.008$. Robustness to uncertainty reflected at the input comes at a cost as demonstrated by the loss in sensor noise rejection in fig. 7. In general, LTR for the Servo Compensator will degrade the sensor noise rejection capacity of the Kalman filter. A balance between robustness and sensor noise rejection must be reached based on the anticipated operating environment.

Reduction of the compensator must be performed if the controller is to be considered practical due to the high order (forty) and the stiffness of the estimator (model has a number of very fast modes). Anderson and Liu summarize a variety of methods ${ }^{17}$ including the balanced realization technique used bere. Controller reduction for the Servo Compensator consists of reducing the order of the stabilizing inner loop containing the Kalman filter and the state feedback gains shown in fig. 3. For the rocket engine, this approach is quite effective since the engine is initially open loop stable and very little compensation is required to dampen the feedforward term. The Servo Compensator was reduced from forty to seven states without any loss of performance or robustness.

\section{Simulation Results}

The reduced Servo Compensator was evaluated on the nonlinear simulation including actuator dynamics by performing the Max $Q$ maneuver. $\operatorname{Max} Q$ is a downthrust from full power to minimum power for a number of seconds until an upthrust command is given to return to full power. To gain a better appreciation for LTR, a comparison was made between the two controller designs without measurement noise as shown in figs. 8 - 11. Excellent control of chamber pressure is achieved throughout Max $Q$ for both designs as shown in fig. 8. A mixture ratio command of 6.011 is given for both controllers shown in fig. 9. Although the nominal controller achieves slightly tighter control than the controller with loop recovery, a spike in MR at six seconds demonstrates the importance of robustmess to unmodeled dynamics. Recall that the design is performed using a $100 \%$ model and the spike occurred at the $65 \%$ operating point indicating that a certain amount of robustness is required for the $100 \%$ design to perform over all power levels. Turbine temperature control is shown in figs. 10 and 11. Excellent command following is obtained for the given temperature profiles. The profiles shown are similar to the uncontrolled temperatures in the existing Block I controller. For this case, OPFV moved very little while OPOV, FPOV and $\mathrm{CCV}$ follow trajectories similar to Block I. Altemate command profiles may be given for the turbine temperatures resulting in a more active OPFV, however they must be consistent with the thrust requirements. Profiles can be selected to minimize turbine fatigue while allowing the required thrust from the engine.

The impact of LTR on sensor noise rejection is demonstrated in figs. 12 - 15 using a zero mean gaussian noise generator. Sensor noise does affect the chamber pressure in fig. 12, but MR is very sensitive to sensor noise in fig. 13. The nominal controller with a greater degree of sensor noise rejection (fig. 7) outperforms the controller with improved robustness in terms of noise suppression. Similar results are obtained for the turbine temperatures as shown in figs. $14-15$. It might appear by inspection of the plots that the LTR controller must have a much higher bandwidth than the nominal controller. However table 1 shows that eigenvalues for the two controllers are on the same order of magnitude. 
Nominal Controller

$\underline{\text { LTR Controller }}$

$\begin{array}{cc}0.000 & 0.000 \\ 0.000 & 0.000 \\ 0.000 & 0.000 \\ 0.000 & 0.000 \\ -0.0136 & -0.0156 \\ -33.47 & -36.34 \\ -65.77 & -74.00\end{array}$

Table 1 Eigenvalues of Servo Compensators

\section{Conclusions}

A frequency domain design method using singular value inequalities is developed for the Servo Compensator which is one of the fundamental components of an Intelligent Control System for a reusable rocket engine. Loop transfer recovery (LTR) is used to recover the required degree of robustness to uncertainty reflected at the plant input in order to allow for engine component wear and variations in engine builds. LTR suffers from several limitations. This paper shows that these can be circumvented if the commands are not used for state reconstruction and a number of sensors are available for feedback as on the Space Shuttle Main Engine (SSME) for the selection of a square, minimum phase, and invertible realization.

In addition, this paper demonstrates the applicability of the method to control of the SSME. In particular, a simplified nonlinear model of the engine is given based on a linear perturbation design model at one hundred percent power. The linear design is shown to hold over the entire operating envelop during mainstage. The example demonstrates the tradeoff between robustness and sensor noise rejection in the Servo Compensator. A certain degree of robustness is required in all designs, the difficulty is to find the correct balance for each application by adjusting a single scalar parameter.

\section{Acknowledgements}

I would like to thank Dr. Duane Mattern for the numerous technical discussions and helpful insights. I would also like to thank Bob Agular at Rocketdyne for his assistance with the simplified nonlinear dynamic model of the rocket engine. Additionally, I would like to thank Carl Lorenzo and Walter Merrill for the opportunity to perform this work.

\section{References}

1. Lorenzo, C.F. and Merrill, W., "An Intelligent Control System for Rocket Engines: Need, Vision and Issues," IEEE Control Systems Mag., Vol. 11, No. 1, pp. 42 - 6, Jan. 1991.
2. Musgrave, J.L., "Advanced Control Modes for the Space Shuttle Main Engine," 1990 Conf. on Advanced Earth-to-Orbit Propulsion Tech. MSFC, Alabama, May 15-17, 1990.

3. Athans, M., "On the Design of PID Controllers Using Optimal Linear Regulator Theory," Automatica, Vol. 7, pp. $643-7,1971$.

4. Davison, E.J., and Goldenberg, A., "Robust Control of a General Servomechanism Problem: The Servo Compensator," Automatica, Vol. 11, pp. 461-471, Sept. 1975.

5. Davison, E.J., "Multivariable Tuning Regulators: The Feedforward and Robust Control of a General Servomechanism Problem," IEEE Transactions on Automatic Control, Vol. AC-21, No. 1, pp. 35-47, Feb. 1976.

6. Wang S., and Munro, N., "An Optimal Design Approach for the Robust Controller Problem," International Journal of Control, Vol. 38, No. 1, pp. 61-85, 1983.

7. Doyle, J.C. and Stein, G., "Robustness with Observers," IEEE Transaction on Automatic Control, Vol. AC - 24, No. 4, pp. 607 - 611, August 1979.

8. Doyle, J.C. and Stein, G., "Multivariable Feedback Design: Concepts for a Classical/Modem Synthesis," IEEE Trans. on Automatic Control, Vol. AC-26, No.1, pp. 4-16, Feb. 1981.

9. Doyle, J.C., "Multivariable Design Techniques Based on Singular Value Generalizations of Classical Control," AGARD Lecture Series 117, Oct. 1981.

10. Freudenberg, J.S, Looze, D.P. and Cruz, J.B., "Robustness Analysis Using Singular Value Sensitivities," International Journal of Control, Vol. 35, pp. 95-116, 1982.

11. Stein, G. and Athans, M., "The LQG/LTR Procedure for Multivariable Feedback Control Design," IEEE Trans. on Automatic Control, Vol. AC-32. pp. 105114, Feb. 1987.

12. D.B. Ridgely, and Banda, S.S., "Introduction to Robust Multivariable Control," Dept. Air Force, Fight Dynamics Lab, Wright-Patterson AFB, Tech. Rep. AFWAL-TR-85-3102, Sept. 1985.

13. Athans, M. and Stein, G., "How to Adapt LQ Regulator Designs to Command-Following and Output-Disturbance Rejection Feedback Control 
Systems," Lecture Notes for 6.232 Multivariable Control Systems, Ref. No. 840418/6232, April 1984.

14. Stein, G. and Doyle, J. "Beyond Singular Values and Loop Shapes," Journal of Guidance and Control, Vol. 14, No. 1, Jan/Feb 1991, pp. 5-16.

15. Matson, C.L. and Maybeck, P.S., "On an Assumed Convergence Result in the LQG/LTR Technique," IEEE Trans. on Automatic Control, Vol. 36, No.1, pp. 123 - 125, Jan. 1991.

16. Nguyen, D.G. "Engine Balance and Dynamic Model," Rocketdyne Div. Rockwell International Corp. Report RL-00001, Version G. \& H. 1981, R(s) 1985.

17. Anderson, Brian D. O., and Liu, Yi, "Controller Reduction: Concepts and Approacbes," IEEE Transactions on Automatic Control, Vol. 34, No. 8, pp. 802 - 11, August 1989.

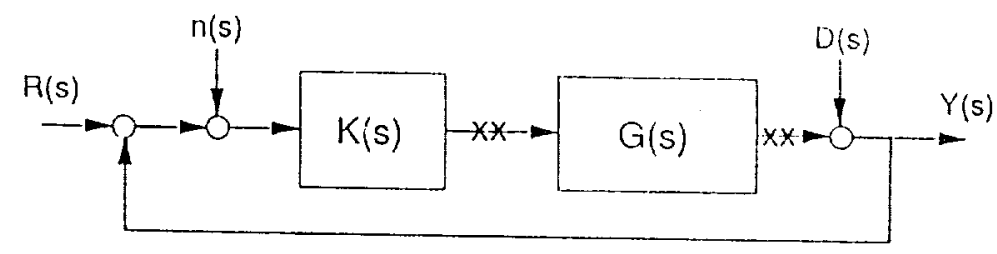

Figure 1 Typical Feedback Selup

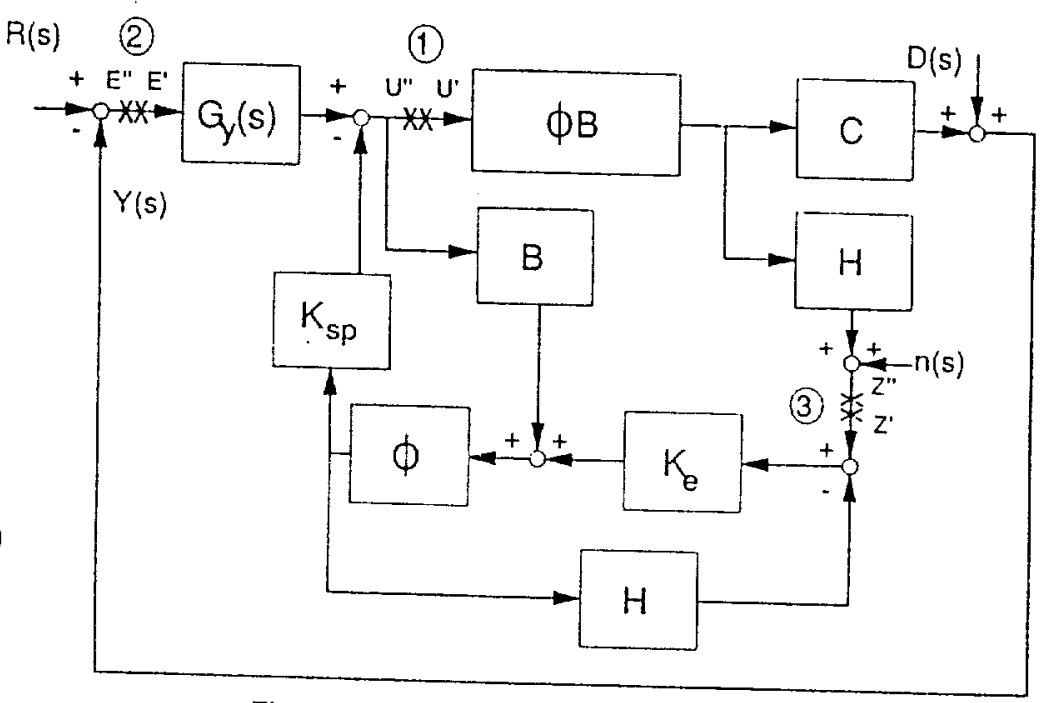

Figure 3 Servo Compensator with Kalman Filter

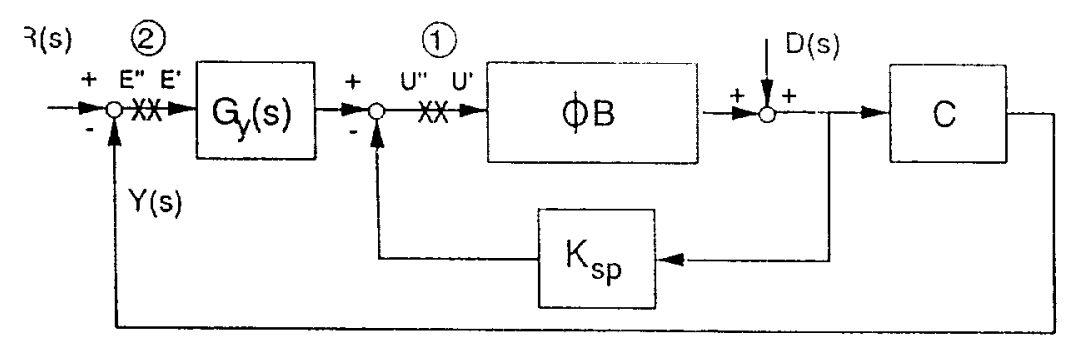

Figure 2 Servo Compensator with Full Stale Feedback

\section{- Sensors \\ 舟 Actuators}

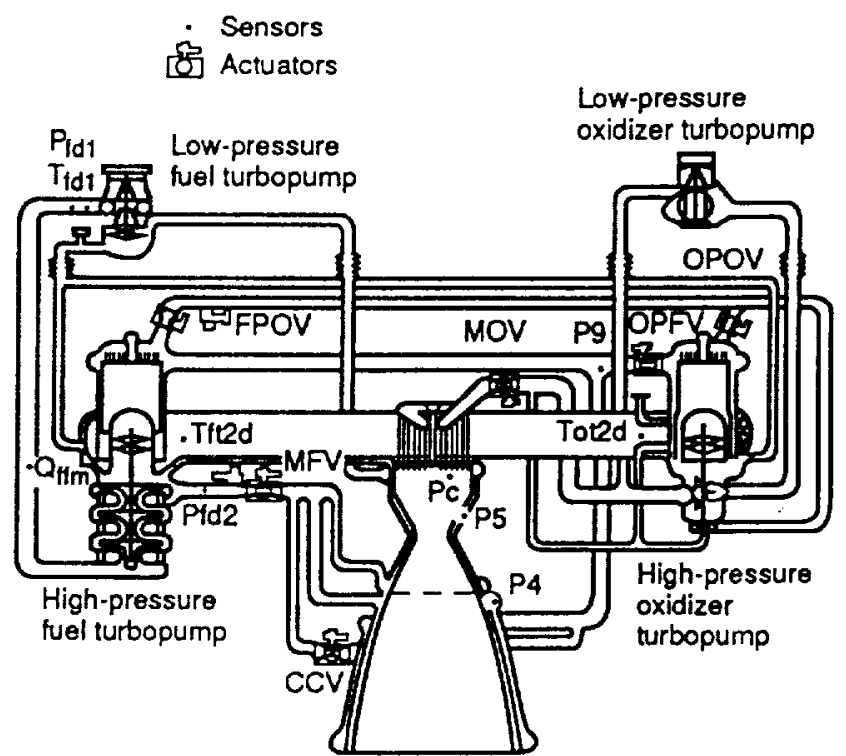

Figure 4. Space shuttle main engine schematic showing engine control valve locations and sensors. 


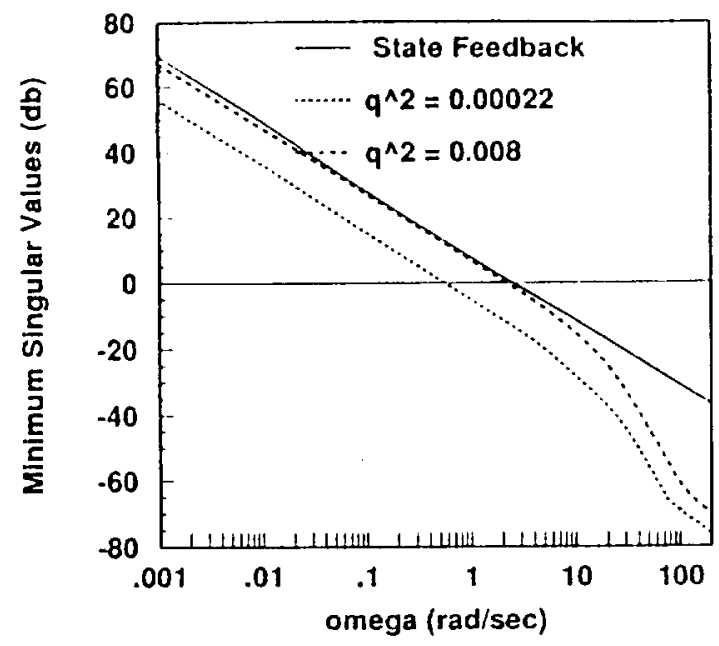

Figure 5 Singular Values with Loop Broken at Input

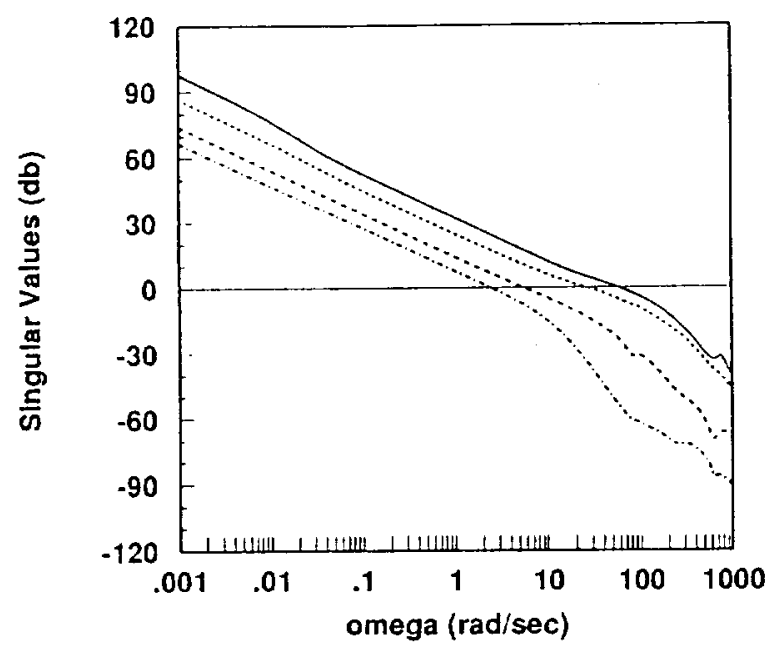

Figure 6 Singular Values of Loop Broken at E(s)

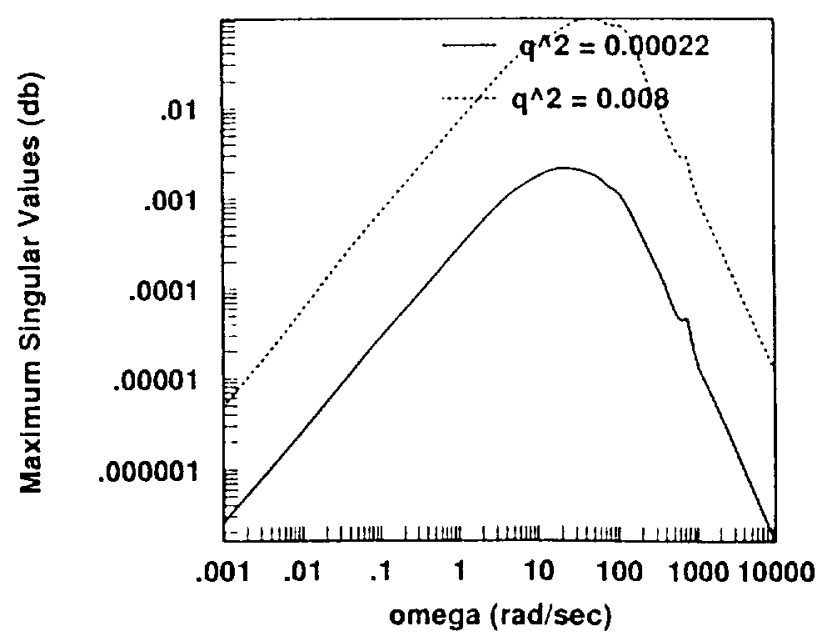

Figure 7 Singular Values for Sensor Noise Rejection

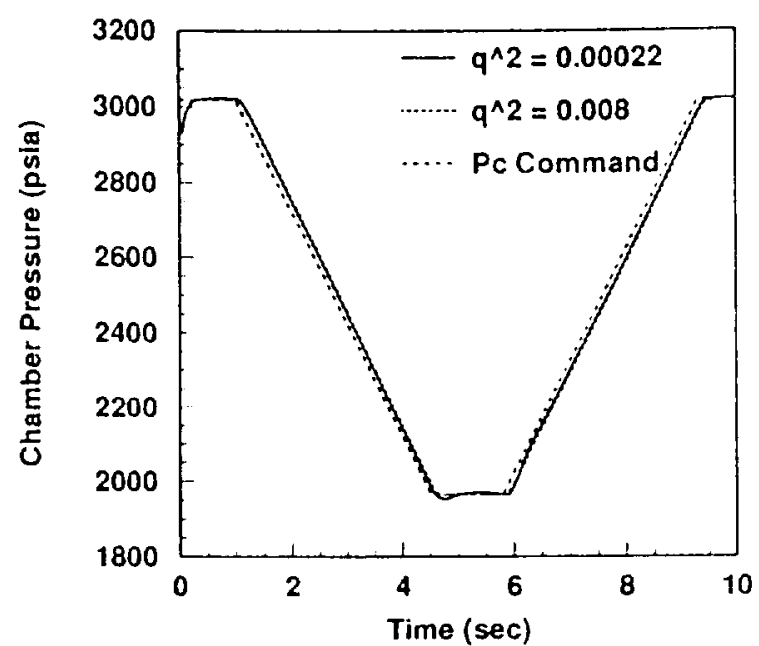

Figure 8 Servo Control of Pc through MAX O

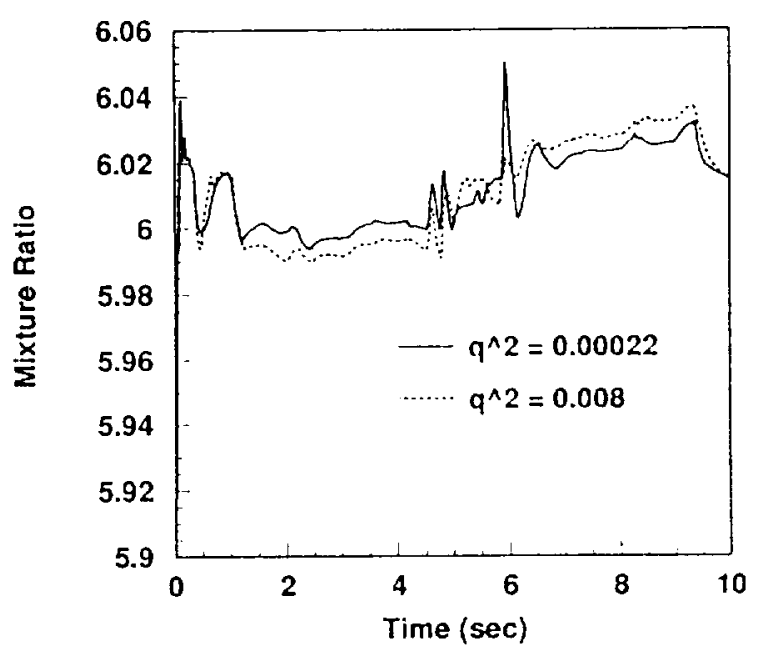

Figure 9 Servo Control of MR through MAX $Q$

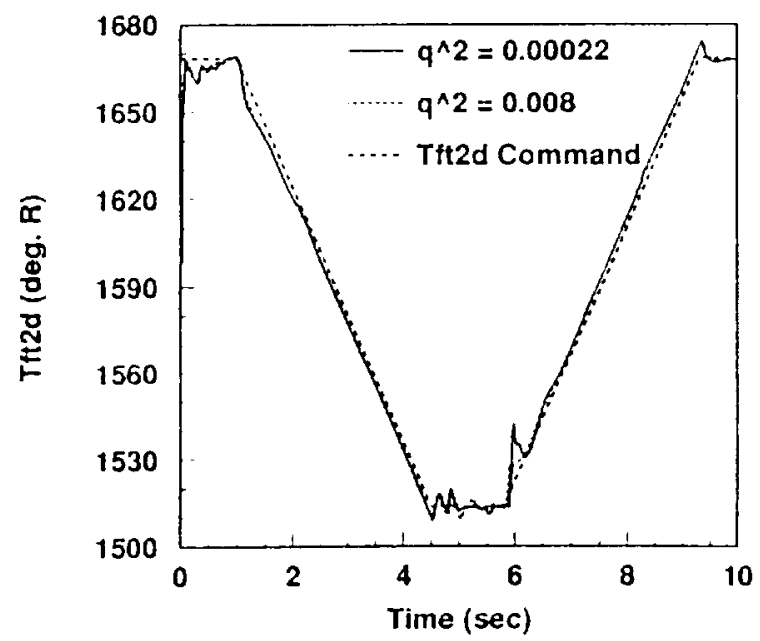

Figure 10 Servo Control of Tit2d through MAX Q 


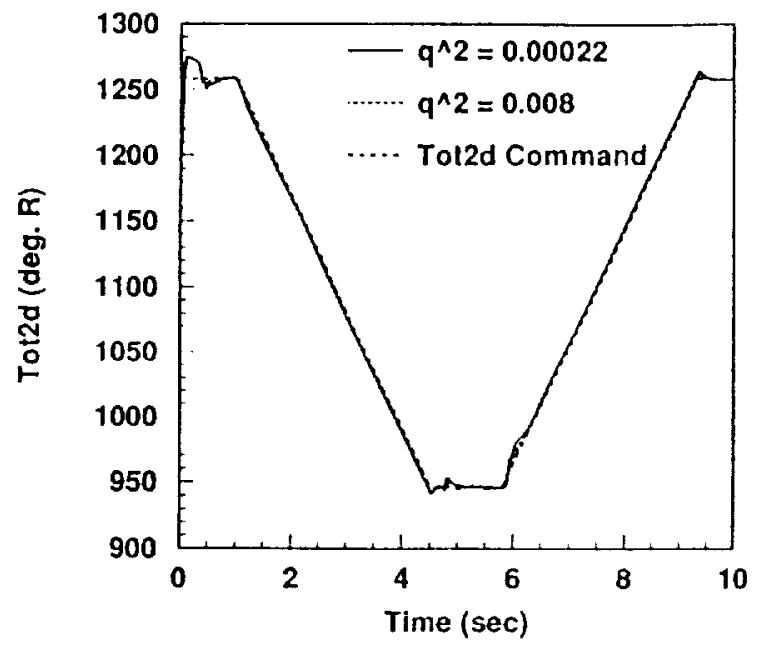

Figure 11 Servo Control of Tot2d through MAX Q

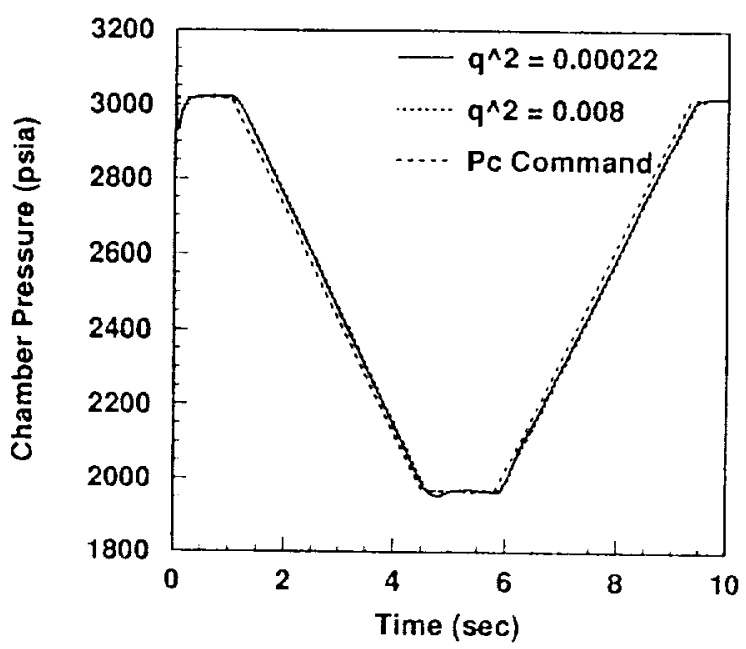

Figure $12 \mathrm{Pc}$ Control with Sensor Noise

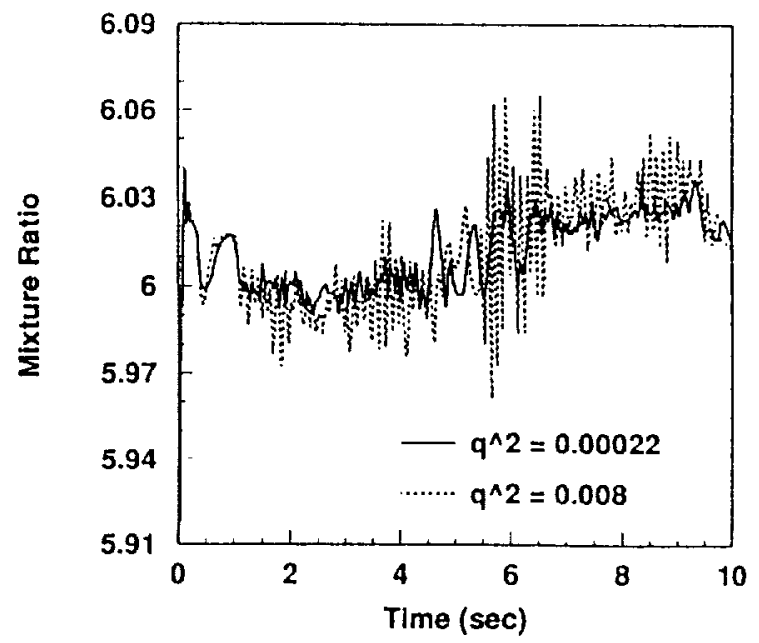

Flgure 13 MR Control with Sensor Noise

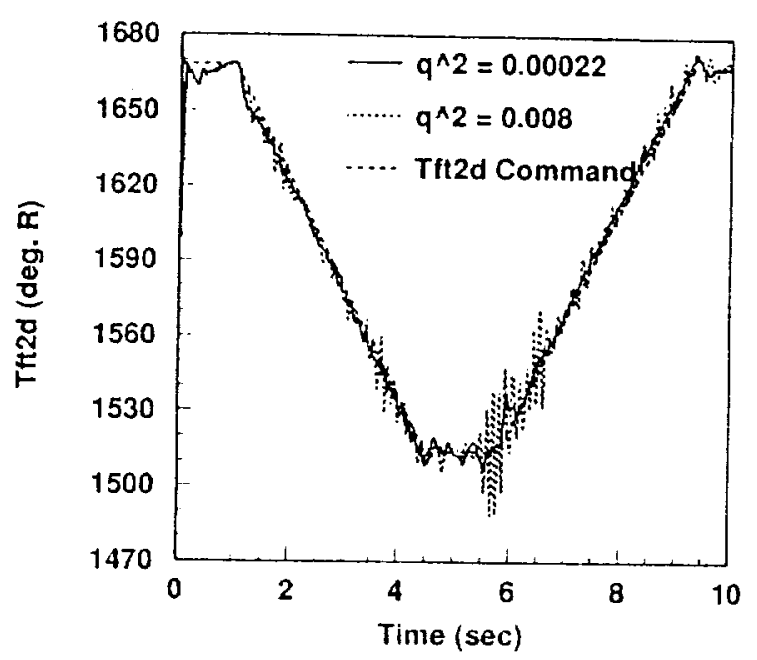

Figure $14 \mathrm{Tft} 2 \mathrm{~d}$ Control with Sensor Noise

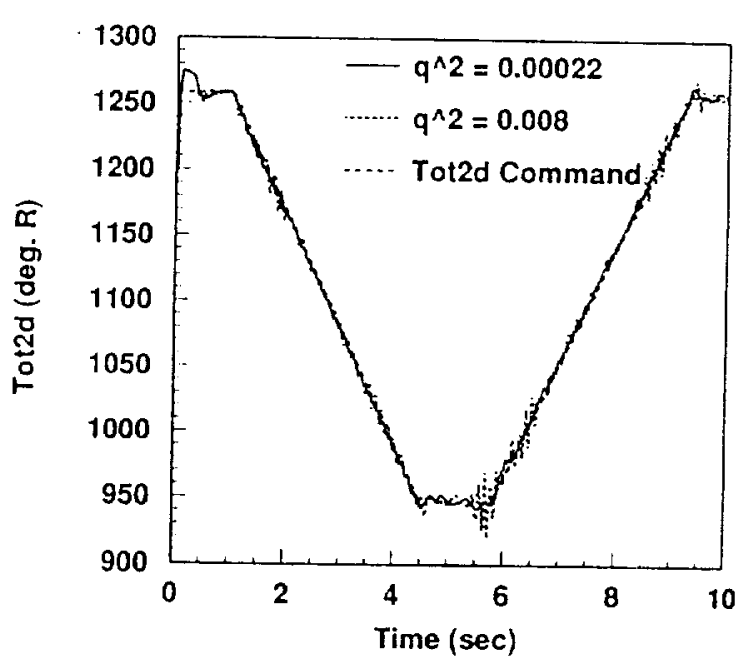

Figure 15 Tot2d Control with Sensor Noise 


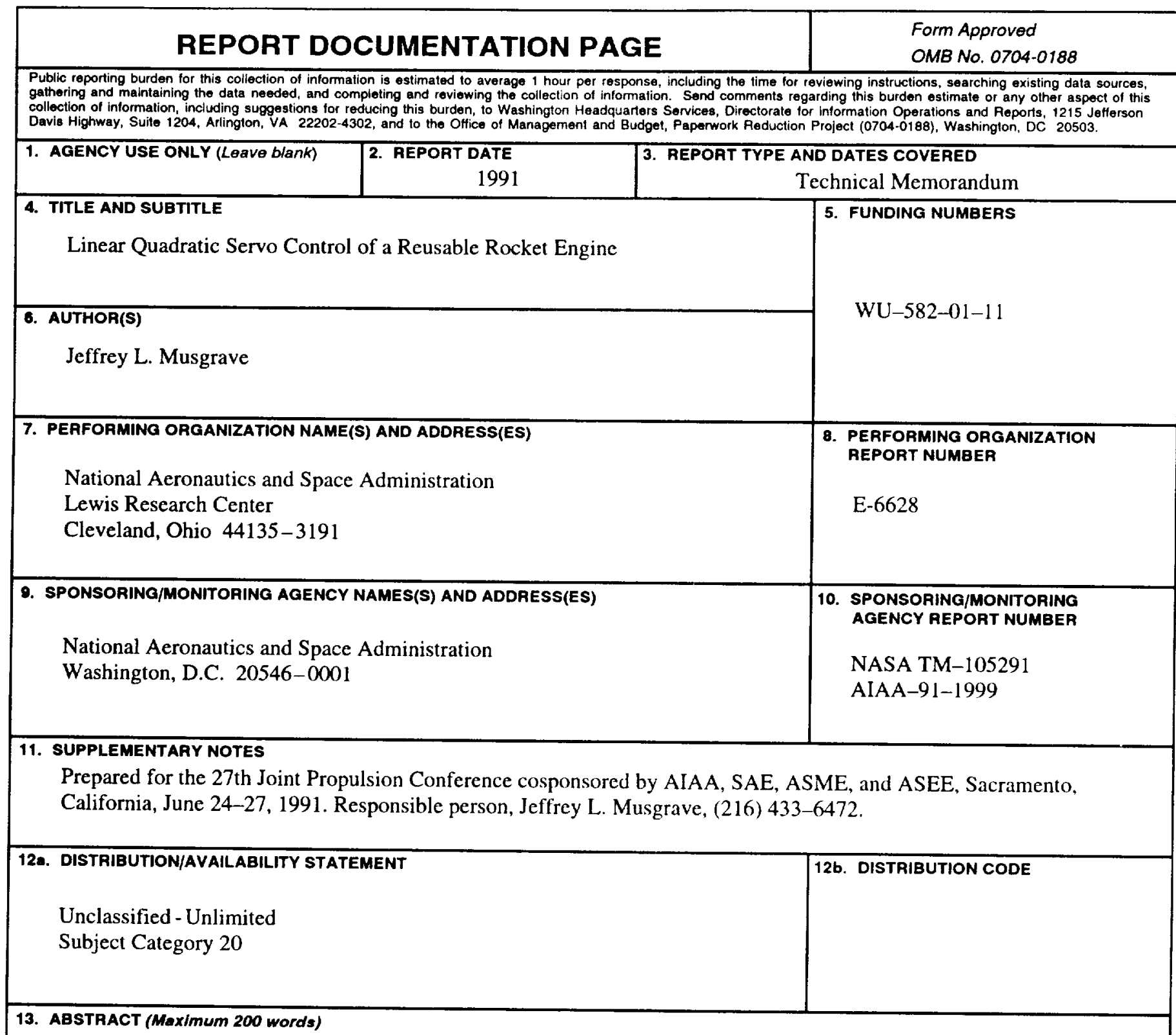

This work develops a new design method for the Servo Compensator in the frequency domain using singular values and applies the method to a reusable rocket engine. An Intelligent Control System for reusable rocket engines has been proposed which includes a diagnostic system, a control system and an intelligent coordinator which determines engine control strategies based on the identified failure modes. The method provides a means of generating various linear multivariable controllers capable of meeting performance and robustness specifications and accommodating failure modes identified by the diagnostic system. Command following with set point control is necessary for engine operation. A Kalman filter reconstructs the state while Loop transfer recovery recovers the required degree of robustness while maintaining satisfactory rejection of sensor noise from the command error. The approach is applied to the design of a controller for a rocket engine satisfying performance constraints in the frequency domain. Simulation results demonstrate the performance of the linear design on a nonlinear engine model over all power levels during mainstage operation.

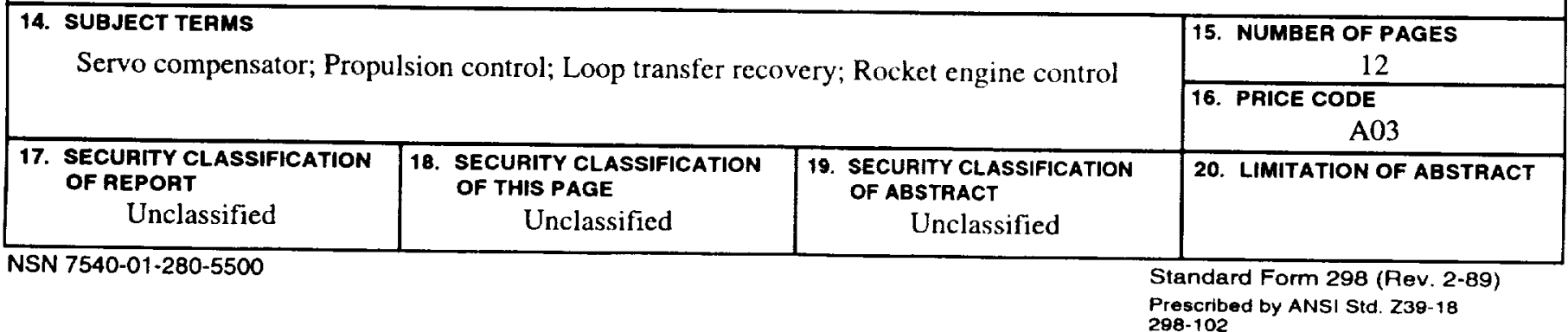


